SMALL BOWEL

\title{
Longitudinal mechanical tension induces growth in the small bowel of juvenile rats
}

\author{
S D Safford, A J Freemerman, K M Safford, R Bentley, M A Skinner
}

See end of article for authors' affiliations

......................

Correspondence to:

Dr S D Safford, PO Box

31295, Duke University

Medical Center, Durham,

NC 27710, USA;

saffo001@mc.duke.edu

Revised version received

3 February 2005

Accepted for publication

10 March 2005

Published online first

19 April 2005

\begin{abstract}
Introduction: The aim of our study was to apply longitudinal force to the small bowel to increase the length of intestine in juvenile rats.

Methods: Fifty juvenile rats had double barrelled, blind loop ostomies created using an isolated segment of bowel. Our intestinal lengthening device was inserted into one of the loops and the second loop served as a control. Once the device was deployed, the experimental, control, and in situ segments of bowel were evaluated for length, weight, histology, and disaccharidase enzyme activity.

Results: Mechanical tension increased intestinal length by 149\%. The lengthened bowel also exhibited a greater total weight $(218 \%)$, greater mucosal weight $(122 \%)$, and increased protein mass (164\%) compared with the control limb of bowel. Histologically, there was a markedly increased thickness of the muscularis propria in the lengthened bowel (200\% increase compared with the control limb). Functionally, we found increased total disaccharidase activity in the lengthened bowel (between $47 \%$ and $350 \%$, depending on the particular enzyme tested; $p<0.01$ ).

Conclusion: Mechanical tension induces intestinal growth by increasing length, weight of the bowel and mucosa, and protein mass. Histological changes, such as increases in Paneth cells, suggest that increased proliferation and reorganisation of the mucosa and muscularis propria are a response to mechanical tension. Functionally, increased intestinal length corresponds with increased disaccharidase activity, thus implying potential increased absorptive capacity of the lengthened bowel.
\end{abstract}

S hort bowel syndrome (SBS) affects between 12 and 120 people per million per year. ${ }^{1}$ In children, the most common cause of SBS is necrotising enterocolitis. The pathology of SBS includes both the total length of bowel removed as well as the function of the remaining segment of bowel. Treatment of SBS involves delivery of nutrients parenterally, surgical attempts at creating length of bowel, and small bowel transplantation. These therapies have had variable success but also are associated with a significant number of complications; therefore, alternative treatments for SBS need to be developed.

We hypothesise that application of tension to the intestine induces growth, resulting in intestinal lengthening. Sufficient lengthening could increase the absorptive area of the intestine such that individuals with SBS could satisfy their nutritional needs through diet alone. Several lines of evidence from other disease states suggest that mechanical tension applied to tissue can in fact induce growth.

Our hypothesis assumes that intestinal tissues are constantly adapting to their surrounding environment. In fact, human intestine does change in response to the nutritional requirements of the body. ${ }^{23} \mathrm{~A}$ number of humoral factors, including insulin-like growth factor, epidermal growth factor, transforming growth factor, and enteroglucagon, have been implicated in some of the adaptive responses of the intestine.

In this study, we created and used a surgically implanted device to place longitudinal tension on a segment of bowel. We hypothesised that application of longitudinal tension would create intestinal length and mucosal growth. We tested our hypothesis in a juvenile rat model in which we created a double barrelled blind loop ostomy and placed tension on one limb. Our data support the potential role of tension to induce growth in the human intestine.

\section{METHODS}

Intestinal lengthening procedure

The study was approved by the Institutional Animal Care and Use Committee at Duke University Medical Center. Fifty juvenile (35-37 days) male CD IGS rats underwent the intestinal lengthening procedure (Charles River Laboratories, Wilmington, Massachusetts, USA). After adequate anaesthesia was attained using a 1:10 dilution of a ketamine/xylazine mixture, a midline laparotomy was made and a $1.5 \mathrm{~cm}$ segment of intestine was isolated approximately $10 \mathrm{~cm}$ distal to the ligament of Treitz (fig 1A). Each end of the isolated segment of intestine was closed using a clip. In the centre of the limb of bowel an opening was created for the opening of the ostomy. This segment of bowel was placed through the lateral abdominal wall and a double barrel ostomy was created (fig 1B). The lengthened and unlengthened segments of bowel were therefore outside of continuity of the normal gastrointestinal stream and were not exposed to any intraluminal nutrition (fig lC). Bowel continuity was created where the isolated segment of bowel was resected. To serve as a control for animal growth, a $1 \mathrm{~cm}$ section of bowel distal to the anastomosis was marked using two prolene stitches measured $1 \mathrm{~cm}$ apart and designated in situ bowel (fig lA).

After creating the double barrel ostomy, our intestinal lengthening device was placed into one limb of the bowel that was designated experimental or lengthened limb and the second limb that served as the control was named control or unlengthened limb (fig 2). Once the device was secured using prolene stitches to the external oblique fascia, the animal was allowed to recover. Animals were placed on a soft diet

Abbreviations: SBS, short bowel syndrome; TGF- $\beta 1$, transforming growth factor $\beta 1$ 

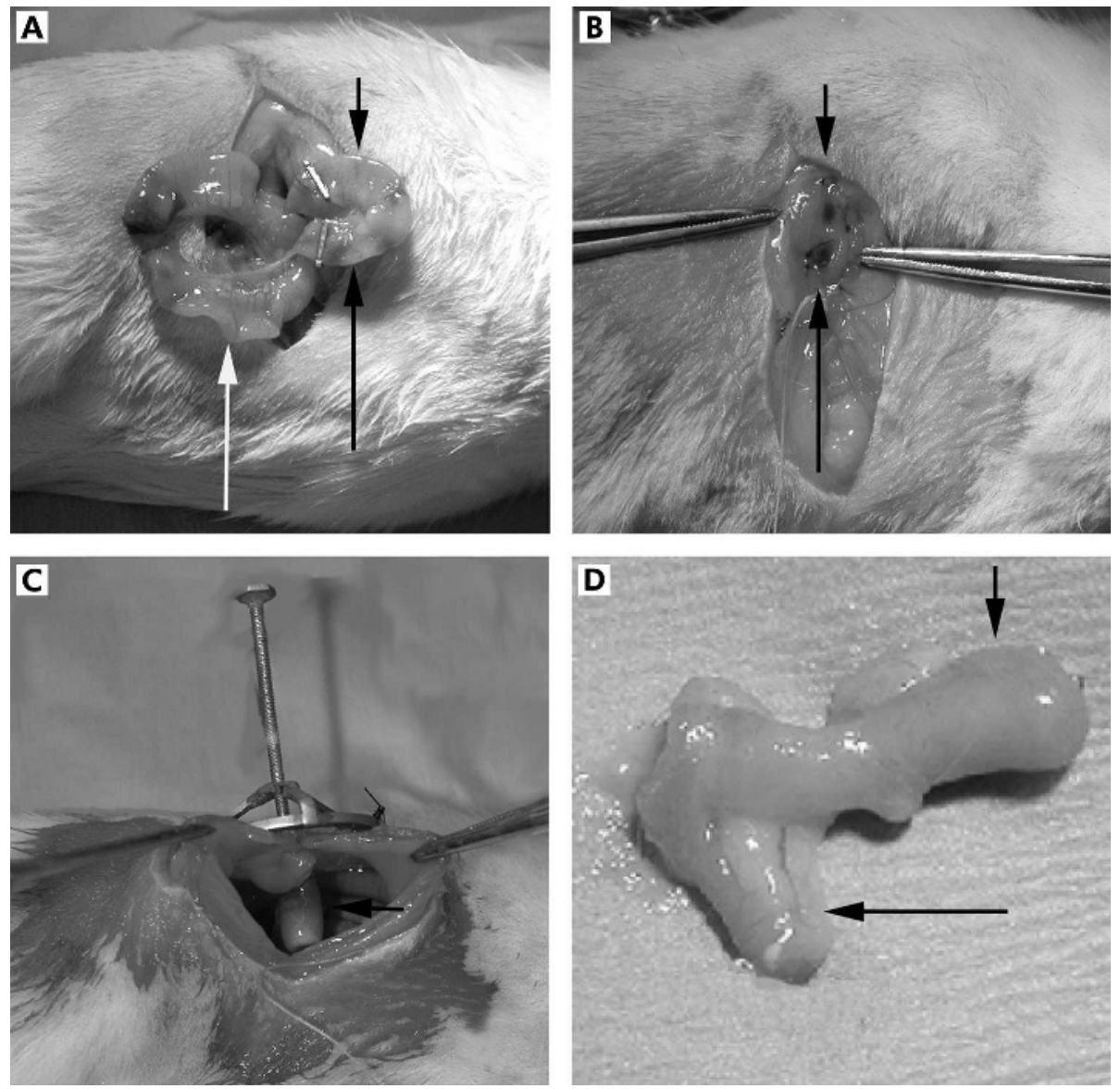

Figure 1 Surgical placement of the intestinal lengthening device. (A) A segment of small bowel was isolated, and an enterotomy was placed in the middle of the isolated loop of bowel. For these photos, the long black arrow indicates the unlengthened limb, the short black arrow the lengthened limb, and the white arrow identifies the in situ bowel. (B) A double barrel ostomy was created with one loop for placement of the intestinal lengthening device, and the second as an internal control. (C) Intestinal lengthening device within the loop of bowel. (D) After 30 days, the isolated limb of small bowel is removed.

postoperatively, and once they passed stools, the device was lengthened at $1 \mathrm{~mm}$ per day. Four animals were sacrificed in the first two days after surgery owing to bowel obstructions.

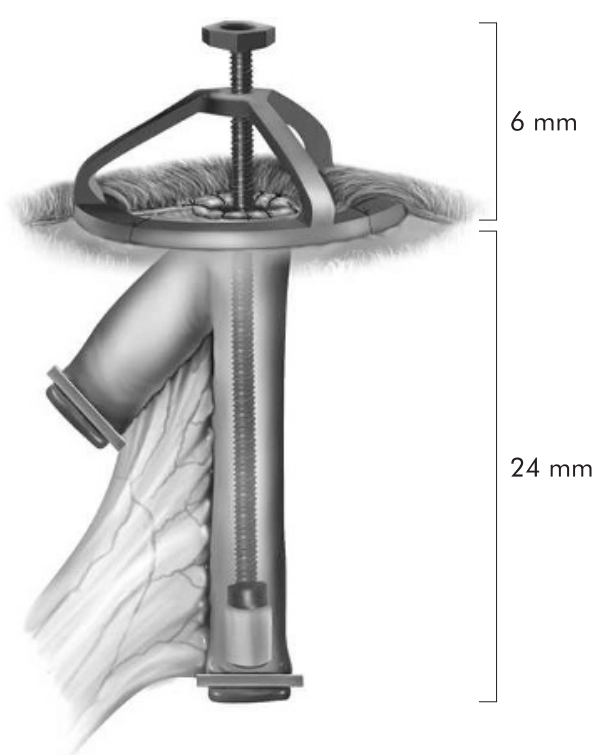

Figure 2 Schematic diagram of the intestinal lengthening device.
After 30 days the animals were sacrificed and measurements were made of lengthened, unlengthened, and in situ segments of bowel (fig 1D). Growth was measured by gross length and weight, and histological measurements included total mucosal thickness, villous height and width, muscularis propria thickness, and serosa thickness. All animals underwent gross measurement of weight and length of the unlengthened, lengthened, and in situ segments. Two measurements by independent observers were made for the segments of bowel. Firstly, the lengthened limb of bowel was measured on tension with the device in place. Secondly, after sacrifice of the animal, the intestinal segment was removed and a measurement was taken with no tension applied. Histological data were determined in 25 animals. In an additional six animals, we assessed the longevity of the intestinal growth. In this group of six animals, the devices were lengthened over a 30 day period, after which the devices remained deployed for another 30 days. After this, the device was unscrewed and intestine remained off tension for another 30 days.

\section{Histological evaluation}

In 25 animals, histological evaluation was performed. For these animals, the mid portion of the bowel was embedded in paraffin and underwent haematoxylin and eosin staining. ${ }^{4}$ Stained slides were then evaluated by an independent pathologist who was blinded to the experimental segment of bowel for number of Paneth cells. Additionally, total 
Table 1 Assessment of growth by measurement of bowel weight, mucosal weight, and enzyme activity of lengthened loff tension), unlengthened, and in situ bowel

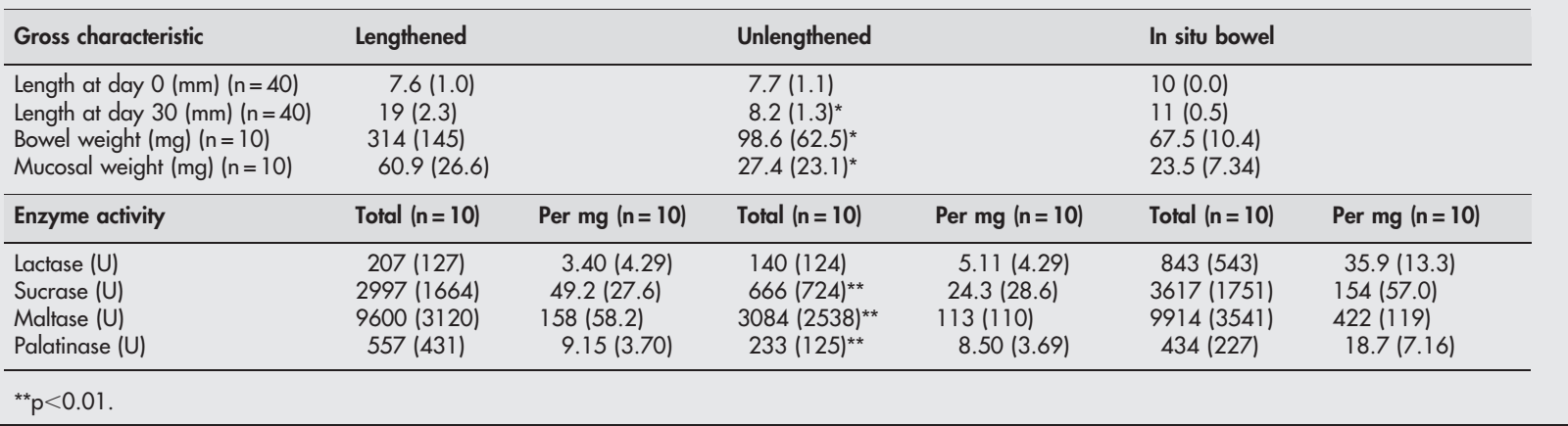

mucosal thickness, villous height and width, muscularis propria thickness, and serosa thickness were determined for each segment. Three measurements were made for each sample and the average was used as the value for that sample.

\section{Protein assay}

Intestinal segments were measured for length, bowel weight, and mucosal weight. Additionally, total protein was measured using a standard protocol of creating a standard protein concentration and measuring UV absorbance at $280 \mathrm{~nm}$. Samples that were sent for later enzymatic evaluation were diluted 1:20 and measured against the standard concentrations of albumin.

\section{Enzyme assay}

As a method to determine the absorptive capacity or function of the lengthened small bowel, enzyme activity and mucosal weight were determined in 10 animals. The segments of lengthened, unlengthened, and in situ bowel were isolated and the mucosa was scraped off the underlying muscularis propria and immediately placed on dry ice. We confirmed complete removal of the mucosa by evaluating the residual intestine by histological staining. These samples were then sent for analysis at Joli Diagnostics Laboratory (Williamsville, New York). Intestinal enzymes assayed included lactase,

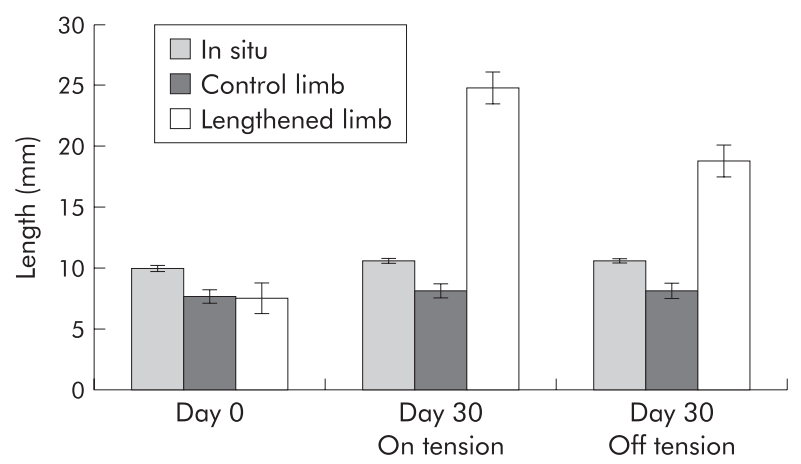

Figure 3 Mechanical tension lengthens the small bowel. These data represent measurements on day 0 and day 30 . The "on tension" group represents measurement of the lengthened intestine with the device deployed, and the "off tension" group demonstrates the size of the lengthened limb of bowel with the device removed. At day 0 , a $15 \mathrm{~mm}$ limb of bowel is opened to create two limbs, the experimental lengthened bowel and the control unlengthened bowel. The in situ bowel was used as a control for normal growth of the animals. The average length of bowel was not different between the lengthened and unlengthened bowel $(7.6$ (1) $\mathrm{mm} \vee 7.7$ (1.1) mm; $\mathrm{p}>0.5, \mathrm{n}=40$ ). After 30 days, the average length of bowel was increased by $228 \%$ with the device on tension, and by $148 \%$ once the device had been removed ( $p<0.01$ ). sucrase, maltase, and palatinase. In brief, mucosal tissue was homogenised and incubated with the appropriate disaccharide substrate. Enzyme hydrolysis resulted in the formation of glucose. A quantitative measurement was then done on the glucose yielded. We analysed the data comparing enzyme activity per milligram of protein and enzyme activity per millimetre of bowel length.

\section{Statistical analysis}

All statistics were analysed using StatsDirect (CamCode, Cambridge, UK) statistical analysis software. We compared the lengthened, unlengthened, and in situ measurements using two way ANOVA. We defined statistical significance as a $\mathrm{p}$ value $<0.01$.

\section{RESULTS}

\section{Intestinal lengthening and growth}

To evaluate both increased length and growth of intestine, we measured total length, bowel weight, and mucosal weight of the lengthened limb of bowel, the unlengthened limb (control) of bowel, and an in situ segment of bowel. The unlengthened segment served as a control for bowel that had undergone surgical manipulation and was not being fed enterally. The in situ segment of bowel represented normal bowel and controlled for normal animal growth. At day 0 , mean lengths of the lengthened, unlengthened control, and in situ segment were 7.6 (1.0) $\mathrm{mm}, 7.7$ (1.1) $\mathrm{mm}$, and 10 (0.0) $\mathrm{mm}$, respectively (table 1 , fig 3 ). After 30 days of increasing the length of our device by $1 \mathrm{~mm}$ per day, the lengthened limb of bowel increased to 24.8 (2.8) $\mathrm{mm}$ with tension, and once the tension was removed, the final length of bowel was $18.8(2.3) \mathrm{mm}$, a $149 \%$ increase in length $(\mathrm{p}<0.01)$. After 30 days, the unlengthened and in situ segments of bowel demonstrated no difference in length

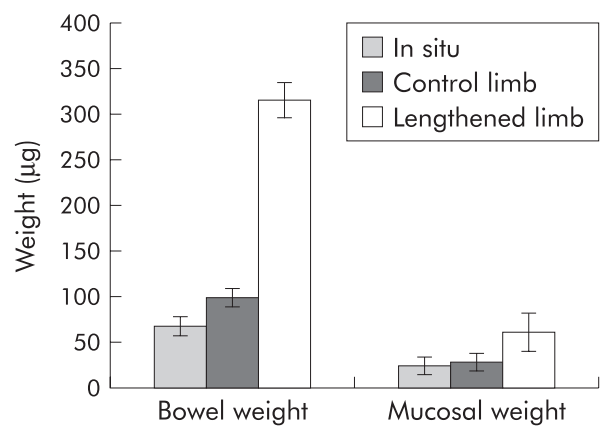

Figure 4 Measurement of weight of the entire bowel and mucosa of in situ, control limb, and lengthened limb. Mucosa from the lengthened limb of bowel was $121 \%$ heavier than that from the unlengthened limb of bowel (60.9 (26.6) mg v $27.4(23.1) \mathrm{mg} ; \mathrm{p}<0.01, \mathrm{n}=10)$. 
$(p>0.01)$. Of note, four of the animals developed bowel obstructions on days $1-3$ of the procedure. Two of the animals developed leaks at the anastomosis where the segment of bowel was removed, and the other two developed tight strictures at the anastomosis secondary to technical difficulties while creating the anastomosis.

In addition to length, we assessed growth by comparison of bowel and mucosal weight. These data were collected only after the animal had been sacrificed and the segments of bowel were removed. Total intestinal weight of the lengthened limb of bowel was $218 \%$ heavier than the unlengthened limb (table 1, fig 4). For mucosal weight, the mucosa from the lengthened limb of bowel was $121 \%$ heavier than the mucosa from the unlengthened limb of bowel (60.9 (26.6) $\mathrm{mg} v 27.43(23.1) \mathrm{mg} ; \mathrm{p}<0.01)$. The increase in mucosal weight was in similar proportion to the increase in length. Additionally, to confirm growth of tissue, we measured an increase in total mucosal protein comparing the unlengthened limb to the lengthened limb of bowel (unlengthened $1.22(0.23) \mathrm{mg} v$ lengthened 2.01 (0.5) mg; $\mathrm{p}<0.01)$. These data support the hypothesis that tension induces mucosal growth with the increase in segmental bowel length. Of note, the total mucosal protein of the in situ bowel was 2.15 (0.4) $\mathrm{mg}$; whereas this was similar in amount to the lengthened bowel, the in situ bowel was significantly smaller and thus we had protein loss secondary to starvation when controlled for length of bowel.

In order to assess whether the mucosa was similar to normal intestinal mucosa, we compared the weight of mucosa per unit length of bowel. The lengthened limb of bowel had no difference in mass of mucosa per length of bowel than the in situ bowel $(3.23 \mathrm{mg} / \mathrm{mm} v 2.4 \mathrm{mg} / \mathrm{mm}$; $\mathrm{p}>0.05)$.

To evaluate the longevity of the intestinal changes, we performed the lengthening procedure on an additional group

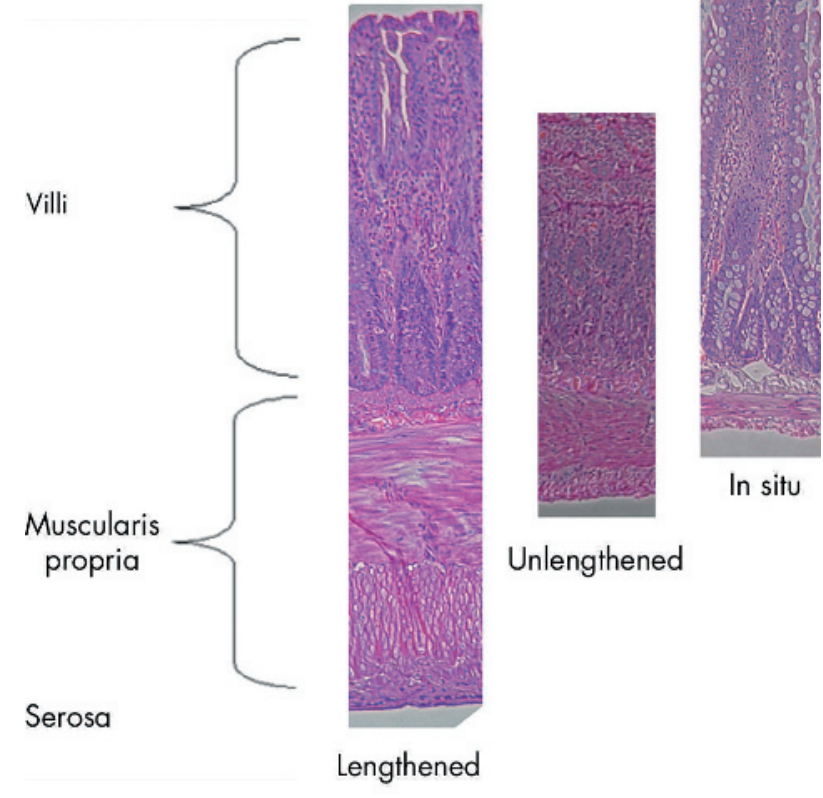

Figure 5 Histology of the lengthened, unlengthened, and in situ small bowel. There were no differences between the lengthened and unlengthened limbs of bowel. The in situ bowel villi were longer than both the lengthened and unlengthened limbs. The lengthened limb of bowel was thicker than both the unlengthened and in situ limbs of bowel. Measurements were taken at $200 \times$ magnification. of animals for which the device was allowed to remain deployed after the lengthening procedure. The lengthened intestine was taken off tension for an additional 30 days to establish whether the increased length was maintained. After the 30 day period off tension, the lengthened segment of bowel was slightly shorter compared with the initial experimental lengthened limb from the first portion of this experiment (15.7 (3.2) mm $v 18.8(2.3) \mathrm{mm}$; $\mathrm{p}=0.02)$ but still lengthened compared with the control unlengthened limb of bowel (15.7 (3.2) mm $v 7.1$ (1.4) mm; $\mathrm{p}<0.01)$. The weight of both the bowel (lengthened 277 (121) mg $v$ unlengthened 92.1 (47) mg; $\mathrm{p}<0.01$ ) and mucosa (lengthened 72 (39.2) mg $v$ unlengthened $31.2(21.1) \mathrm{mg} ; \mathrm{p}<0.01$ ) also remained increased compared with our control unlengthened segment of bowel.

\section{Histological measurements}

To identify what cellular changes were occurring in response to the external tension, we evaluated the basic histological components of the intestine, including total mucosal thickness, total muscular thickness, villous height, villous width, and serosal width. Most remarkable was the marked muscularis propria thickening in the lengthened bowel (fig 5). Lengthened bowel muscularis propria was $210 \%$ and $740 \%$ thicker than unlengthened and in situ bowel, respectively (table 2 ). We did not identify any significant difference in total mucosal height, villi width or height, or serosal thickness between lengthened bowel and in situ bowel; hence the lengthened segment of bowel exhibited similar histological measurements compared with the normal intestine. Whereas no significant difference was identified, both the lengthened and unlengthened limbs of bowel tended towards decreased measurements of villi. These findings are consistent with the expected atrophy of the villi owing to the absence of enteral nutrition to the isolated segments of bowel.

An additional observation was the significant increase in Paneth cells that were found in the lengthened limb of bowel. In each crypt there was an average of 5.3 cells per crypt in the lengthened limb of bowel versus 1.4 cells/crypt in the unlengthened control $(\mathrm{p}<0.01)$. Similar to the unlengthened controls, the average number of Paneth cells in the in situ bowel was 1.7 cells/crypt.

\section{Enzyme activity}

We evaluated function and confirmed growth of the lengthened limbs of bowel by assessing the enzymatic activity that was present in the mucosa of the lengthened, unlengthened, and in situ limbs of bowel (table 1). The in situ segment of bowel remained in continuity with the normal intestinal contents and was therefore used as the control for normal functioning bowel. To standardise enzyme activity, we measured enzyme activity per milligram of protein. Compared with both the lengthened and unlengthened limbs of bowel, in situ bowel had significantly greater enzyme activity per mg protein for all enzymes studied. For the lengthened limb of bowel that demonstrated doubling of both enzymatic activity and total protein, there was no difference between the lengthened and unlengthened limbs of bowel for enzyme activity per milligram protein.

After normalising for enzyme activity per milligram of tissue and demonstrating no difference between lengthened and unlengthened limbs of bowel, we evaluated total enzyme activity for lengthened, unlengthened, and in situ bowel. We hypothesised that if actual growth had occurred, we should detect an equivalent increase in enzyme activity in addition to increased length. Enzymatic activity for the lengthened limb of bowel was significantly greater for sucrase, maltase, and palatinase (table 1). For sucrase, maltase, and palatinase, the 
Table 2 Histological measurements of lengthened, unlengthened, and in situ bowel

\begin{tabular}{lllc}
\hline & $\begin{array}{l}\text { Lengthened } \\
\text { (n= 25) }\end{array}$ & $\begin{array}{l}\text { Unlengthened } \\
(\mathbf{n = 2 5 )}\end{array}$ & $\begin{array}{c}\text { In situ bowel } \\
\text { ( } \mathbf{n = 2 5 )}\end{array}$ \\
\hline Mucosal thickness $(\mu \mathrm{m})$ & $428(90.2)$ & $377(45.5)$ & $522(131)$ \\
Muscular thickness $(\mu \mathrm{m})$ & $394(43.9)$ & $130.5(35.3)$ & $47.4(10.0)$ \\
Villous height $(\mu \mathrm{m})$ & $340(64.4)$ & $234(43.2)$ & $372(75.3)$ \\
Villous width $(\mu \mathrm{m})$ & $61.4(17.2)$ & $46.3(8.74)$ & $152(27)$ \\
Serosa $(\mu \mathrm{m})$ & $44.7(2.05)$ & $30.5(23.8)$ & $17.2(6.93)$ \\
\hline
\end{tabular}

lengthened limb of bowel had increased enzymatic activity by $350 \%, 211 \%$, and $140 \%$, respectively. We did not identify a significant increase in lactase activity. Despite the lengthened limb of bowel being longer than the in situ bowel, we found no increased enzyme activity; for lactase, the in situ bowel had significantly higher levels of lactase activity. These data further support the hypothesis that the bowel is responding to tension by actual growth and the absorptive area has increased in similar proportion to the increase in length.

\section{DISCUSSION}

Short gut syndrome requiring total parenteral nutrition affects over 200000 patients in the USA and costs \$150 000 per patient annually. ${ }^{56}$ The most common cause of short gut syndrome in children is enterocolitis, wherein large segments of bowel are resected secondary to necrosis. ${ }^{7}$ Surgical removal of greater than $70 \%$ of the small bowel usually leads to short gut syndrome characterised by diarrhoea, malabsorption, malnutrition, and failure to thrive. ${ }^{8}{ }^{9}$ Surgical techniques that have been developed to attempt to slow intestinal transit, increase the absorptive surface, or create length have met with variable success. ${ }^{10}{ }^{11}$ Based on the difficulties with these treatments, other methods to improve length and absorption need to be investigated. Our group has hypothesised that application of mechanical tension would lead to increased length and growth of intestine. To test this hypothesis, we created an intestinal lengthening device to apply tension on an isolated limb of bowel to assess for increased length and weight. This study is the first to successfully demonstrate that tension induces increased length, growth, and increased enzymatic activity in the small intestine.

The principle of tension inducing growth of tissue has been supported in multiple systems. Osteodistraction techniques have lengthened bone at a rate of $1 \mathrm{~mm}$ per day. ${ }^{12}$ Other examples of tension causing tissue growth include plastic surgical techniques of stretching skin over tissue expanders and growth of muscle over tumours. ${ }^{9}$ In the gastrointestinal system, oesophageal lengthening has been proposed through applying tension to close long gap atresias. ${ }^{13}$ Other groups have attempted to elicit growth of intestine through application of traction on the intestine but have either not measured parameters of growth or met with no success. ${ }^{9}{ }^{14}$ In these studies, there has been suggestion of increasing length but none of the studies demonstrated growth or increased enzyme activity. In the study by Printz and colleagues, the device that was used for applying tension was thought to lose traction and thereby not stimulate growth. ${ }^{9}$ In contrast, our group has designed our intestinal lengthening device to apply continuous tension on an isolated segment of bowel. We increased the tension rod by $1 \mathrm{~mm}$ per day; this rate of change was arbitrarily based on length in other tissue models, including bone.

To analyse the effect of tension on the intestine, we measured both the length of the intestine and characterised growth through measurement of bowel weight, mucosal weight, and mucosal protein mass. In our study, we found that the rat intestine increased in length by $151 \%$ over the
30 day period ( 7.5 to $18.8 \mathrm{~mm}$ ). We performed our final measurement only after the lengthening device had been removed. The initial measurement of the lengthened intestine was $24.8 \mathrm{~mm}$ when on traction and the tissue reduced its length after removal of tension. Thus we still maintained tension at the end of the experiment but the final length of intestine was without any external tension.

Once we had consistently shown that tension stimulated an increased length of intestine, we next examined other parameters to elucidate whether the tissue was simply being stretched or if tension was inducing actual growth of tissue. To analyse the growth of tissue, we evaluated increases in intestinal weight, mucosal weight, and mucosal protein mass. Mucosal weight and total protein mass increased by $121 \%$ and $164 \%$, respectively. These changes were similar to the proportional change in intestinal length. Total bowel weight increased by a significantly greater amount (218\%) than intestinal length. These data suggested that other components of the bowel wall were changing at a greater rate than the mucosa. Histologically, the muscularis propria had hypertrophied and most likely accounts for the excess weight gain.

In addition to bowel weight, one would expect that if there were tissue growth, enzymatic activity would also increase by similar amounts. In fact, we found that the lengthened limbs of bowel exhibited more than double the enzymatic activity for all disaccharidases, with the exception of lactase. The loss of lactase is found in other starvation models, and in these models there is a return of enzymatic activity once intraluminal nutrition is restored. ${ }^{15}$

Histologically, the muscularis propria of the experimental lengthened bowel segment demonstrated marked hypertrophy and was $208 \%$ thicker than the control unlengthened limb of bowel. These findings were not specific to the application of tension as both the lengthened and unlengthened limbs of bowel muscularis propria were thicker than in situ bowel. Thus some amount of muscularis propria thickening seemed to be a response of the surgical isolation of the limb of bowel. These findings suggest that both tension and the surgical procedure independently caused a local response to induce muscularis propria thickening. The effects were apparently additive as there was increased muscularis propria hypertrophy in the limb subjected to tension. These changes in the muscularis propria may pose significant problems for future use of the lengthened limb of bowel, and future models may need to address these changes with other mechanisms for applying traction, including peristaltic application of force. These various methods of applying force are the focus of future studies.

Alteration of the histological appearance in response to tension has been demonstrated in a number of other tissue models. Many cells are sensitive to mechanical forces and can change their phenotype and surrounding extracellular matrix in response to changes in their mechanical environment. ${ }^{16}$ For example, mechanical stress in human airway epithelial cells elicits a matrix remodelling response in unstressed cocultured lung fibroblasts through soluble signals. The level 
of response in our system was not measured but in these other tissue models responses were magnitude dependent. ${ }^{16}$ In the future we will need to more finely monitor the amount of tension being applied across the bowel wall and compare the histological changes, specifically muscularis propria thickness, with the level of tension.

Potential mechanisms whereby stretch regulates soluble cytokines and the local matrix in intestinal smooth muscle are suggested by studies demonstrating alterations in signalling pathways and gene expression in response to a single stretch, a change in cell shape, or shear stretch. Tonic stretch enhanced both transforming growth factor $\beta 1$ (TGF$\beta 1)$ and $\alpha \mathrm{l}(\mathrm{I})$ collagen protein and mRNA abundance through increased transcription in intestinal smooth muscle cells in vitro. ${ }^{17}$ In the human intestine, TGF- $\beta 1$ has been shown to be a key regulator of collagen deposition, growth, and wound healing. ${ }^{17}$ In culture, TGF- $\beta 1$ stimulates collagen synthesis through upregulation of collagen, production of protease inhibitors, and suppression of proteolytic enzymes. ${ }^{18} 19$ Additional studies must be performed to determine which molecular factors are responsible for the tension inducing muscularis propria hypertrophy seen in our experiments.

Additional histological observations included the marked proliferation of Paneth cells in the crypts of the lengthened limbs of bowel. Paneth cells are located at the bottom of crypts and are derived from multipotent intestinal stem cells. ${ }^{20}$ The function of these cells is not clear but Paneth cells have been shown to produce regulatory molecules, including growth factors, suggesting these may regulate proliferation and differentiation programmes of epithelia lineages. ${ }^{21}$

Once we had demonstrated the proof of principle of tension increasing length and growth of the intestine, we addressed the question of whether the lengthened bowel would be functional. We approached this question of function indirectly by evaluating the mucosal histological characteristics and enzymatic activity of the lengthened limb of bowel in comparison with the unlengthened control limb. Mucosal thickness was similar for the lengthened and unlengthened limbs of bowel; however, compared with the in situ bowel, both the lengthened and unlengthened limbs of bowel were thinner. This finding was not unexpected as our experimental and control limbs had no enteral nutrition and thus mucosal thinning was expected.22 ${ }^{23}$ Similarly, the villi for both the lengthened and unlengthened bowel were shorter and thinner than the in situ bowel.

Finally, once length and growth were confirmed and our data supported increased function in the lengthened limb of bowel, we confirmed the longevity of the changes that were induced by tension. We found that 30 days after the tension was removed the lengthened intestine was still $114 \%$ greater in length than the original length of the isolated segment of bowel. These data support the notion that the increased length and growth induced by tension on the intestine is sustained once tension has been removed, thereby confirming the durability of the growth.

Whereas we have proven that tension induces length, growth, and an increase in mucosal enzymatic activity, we have not yet proven the true functional capacity of these tissues. The inability to replace the small segment of bowel into normal continuity is a limitation of this experimental model in the rat. The small size of the rat intestine was also important to the bowel obstructions that we observed early in our experience. In the future, we plan on taking this concept to a larger animal in which it will be technically easier to perform the in situ bowel anastomosis and also be feasible to replace the lengthened segment into the enteric stream to document adequate function.
In this study, we report the first successful application of tension to induce length and growth in rat intestine. The increased length and growth were sustained over a 30 day period, even after the tension was removed. Functionally, we have demonstrated increased disaccharidase enzymatic activity in our lengthened limb of bowel. Histological changes occurring in response to tension include muscularis propria hypertrophy and Paneth cell proliferation. These findings have potential importance to the application of this principle in the future treatment of short bowel syndrome.

\section{Authors' affiliations}

S D Safford, A J Freemerman, K M Safford, R Bentley, M A Skinner, Department of Surgery, Division of Pediatric Surgery, Duke University Medical Center, Durham, NC, USA

Conflict of interest: None declared.

This work was presented in part at the American Pediatric Surgical Association in May 2002.

\section{REFERENCES}

1 Vanderhoof J, Young R. Enteral and parenteral nutrition in the care of patients with short-bowel syndrome. Baillieres Best Pract Res Clin Gastroenterol 2003; 17:997-1015.

2 Williamson R. Intestinal adaptation (first of two parts). Structural, functional and cytokinetic changes. N Engl J Med 1978;298:1393-402.

3 Fisher K, Dhanvantari S, Drucker D, et al. Intestinal growth is associated with elevated levels of glucagon-like protein 2 in diabetic rats. Am J Physiol 1997;273:E815-20.

4 Hine I. Block staining of mammalian tissues with hematoxylin and eosin. Stain Technol 1981;56:119.

5 Howard L, Heaphey L, Fleming CR, et al. Four years of North American registry home parenteral nutrition outcome data and their implications for patient management. J Parenter Enteral Nutr 1991;15:384-93.

6 Howard L, Ament M, Fleming CR, et al. Current use and clinical outcome of home parenteral and enteral nutrition therapies in the United States. Gastroenterology 1995;109:355-65.

7 Schalamon J, Mayr J, Hollwarth M. Mortality and economics in short bowel syndrome. Baillieres Best Pract Res Clin Gastroenterol 2003;17:931-42.

8 Shanbhogue LK, Molenaar JC. Short bowel syndrome: metabolic and surgical management. Br J Surg 1994;81:486-99.

9 Printz H, Schlenzka R, Requadt $P$, et al. Small bowel lengthening by mechanical distraction. Digestion 1997;58:240-8.

10 Saday C, Mir E. A surgical model to increse the intestinal absortive surface: Intestinal lengthening and growing neomucosa in the same approach. J Surg Res 1996;62:184-91.

11 Bianchi A. Intestinal loop lengthening $-A$ technique for increasing small intestinal length. J Pediatr Surg 1980;15:145.

12 llizarov G, Devyatov A, Kamerin V. Plastic reconstruction of longitudinal bone defects by means of compression and subsequent distraction. Acta Chir Plast 1980;22:32-41.

13 Foker J, Linden B, Boyle E, et al. Development of a true primary repair for the full spectrum of esophageal atresia. Ann Surg 1997;226:533-43.

14 Chen Y, Zhang J, Qu R, et al. An animal experiment on short gut lengthening. Chin Med J 1997;1 10:354-7.

15 Ortega $M$, Nunez $M$, Suarez $M$, et al. Age-related response of the small intestine to severe starvation and refeeding in rats. Ann Nutr Metab 1996;40:351-8.

16 Swartz M, Tschumperlin D, Kamm R, et al. Mechanical stress is communicated between different cell types to elicit matrix remodeling. Proc Natl Acad SciU SA 2001;98:6180-5.

17 Gutierrez J, Perr H. Mechanical stretch modulates TGF- $\beta 1$ and alphal(I) collagen expression in fetal human intestinal smooth muscle cells. Am J Physiol 1999;277:G1074-80.

18 Edwards D, Murphy G, Reynolds J, et al. Transforming growth factor $\beta$ modulates the expression of collagenase and metalloproteinase inhibitor EMBO J 1987;6:1899-904.

19 Graham M, Bryson G, Diegelmann R. Transforming growth factor- $\beta 1$ selectively augments collagen synthesis by human intestinal smooth muscle cells. Gastroenterology 1990;99:447-53.

20 Darmoul D, Ouellette A. Positional specificity of defensin gene expression reveals Paneth cell heterogeneity in mouse small intestine. Am J Physiol 1996;271:G68-74.

21 Ganz T. Paneth cells: guardians of the gut cell hatchery. Nat Immunol 2000;1:99-100.

22 Martin G, Wallace L, Sigalet D. Glucagon-like peptide-2 induces intestinal adaptation in parenterally fed rats with short bowel syndrome. Am J Physiol 2004;286:G964-72.

23 Adegoke O, McBurney M, Samuels S, et al. Modulation of intestinal protein synthesis and protease mRNA by luminal and systemic nutrients. Am J Physiol 2003;284: G1017-26. 